\title{
Experiencia inicial del modelo de Educación Deportiva en primero de Educación Primaria. Percepción del alumnado y del docente \\ Initial experience with the Sport Education model in primary school first-grade. Students and teachers' perceptions \\ *Diego Martínez de Ojeda, **Federico Puente-Maxera, **Antonio Méndez-Giménez, ***María Pilar Mahedero- Navarrete \\ *Consejería de Educación. Región de Murcia (España), **Universidad de Oviedo (España), ${ }^{* * * C o n s e j e r i ́ a ~ d e ~ E d u c a c i o ́ n . ~ J u n t a ~ d e ~}$ Andalucía (España)
}

\begin{abstract}
Resumen: A pesar de haber sido aplicado con éxito en estudiantes de diferentes edades, en la actualidad no hay constancia de investigaciones que hayan examinado el impacto del modelo de Educación Deportiva (MED) entre el alumnado de primer curso de Educación Primaria (EP). El objetivo del presente estudio fue implementar una temporada a partir del MED en un curso de primero de EP a efectos de conocer su impacto sobre las percepciones docentes y discentes respecto al propio modelo, así como sus efectos motivacionales (satisfacción de las necesidades psicológicas básicas). Esta cuestión se abordó desde una perspectiva cuantitativa y cualitativa. La muestra se compuso de 18 estudiantes $\left(M_{\text {edad }}=6.61\right.$ ) y un docente (con una experiencia de 12 años, tres de ellos aplicando el MED), pertenecientes a un colegio ubicado en una zona rural del sureste de España. Es un estudio de caso en el que se empleó un diseño cuasi-experimental con medidas repetidas pretest-postest. La percepción discente fue captada a partir de entrevistas, cuestionarios y dibujos, mientras que la percepción docente fue recogida mediante un diario y entrevistas. Los resultados son consistentes con los de estudios previos sobre el MED entre escolares de corta edad. Se encontraron aumentos significativos en los niveles de cultura deportiva y entusiasmo. Igualmente, se observaron mejoras en las NPB. Se sugiere que el MED puede ser aplicado con éxito en primero de EP. Palabras clave: Educación deportiva, educación física, educación primaria, modelo pedagógico, motivación.
\end{abstract}

\begin{abstract}
The Sport Education model has been widely investigated across different ages. Nevertheless, to date there is no published research that examines its impact on primary school first-grade students. The goal was to implement a Sport Education season in a firstgrade class to assess students and teacher' perceptions about the model and its motivational effects (basic psychological needs satisfaction). This question was addressed from a quantitative and qualitative perspective. The sample was composed by 18 students $\left(M_{\text {age }}=6.61\right)$ and one teacher enrolled in a school from a rural area in southeastern Spain. A quasi-experimental design, pretest-postest measures, was carried out. Students' perceptions were collected via questionnaires, interviews, and drawings, while teacher's perspective was gathered with interviews and diary notes. Results are consistent with previous Sport Education studies in early primary years. There were found significant increments on literacy and enthusiasm, as well as improvements on motivational domains. Findings suggest there is potential enough for introducing Sport Education in the whole primary education stage. Most relevant didactical implications are also discussed. Key words: Sport education, physical education, primary education, pedagogical model, motivation.
\end{abstract}

\section{Introducción}

La investigación empírica muestra al modelo de Educación Deportiva (MED, Siedentop, 1994) como un modelo pedagógico con potencial para promover aprendizajes significativos en un amplio espectro de estudiantes (Metzler, 2011). El MED surge con el propósito de ofrecer al alumnado experiencias deportivas más positivas, completas y auténticas (Siedentop, Hastie y van der Mars, 2011). Sus objetivos principales contemplan la formación de deportistas cultos (literate), competentes (competent) y entusiastas (enthusiastic). Partiendo de diferentes propuestas, una amplia producción científica se ha centrado en examinar dichos propósitos (Hastie y Wallhead, 2016) certificando las fortalezas del modelo en el desarrollo técnico-táctico (Hastie, Sinelnikov, y Guarino, 2009; Mahedero, Calderón, Arias, Hastie, y Guarino, 2015), la amistad (García-López, Gutiérrez, González-Víllora, y Valero, 2012), la deportividad (MéndezGiménez, Fernández-Río, y Méndez-Alonso, 2015) o el entusiasmo (Calderón, Martínez de Ojeda y Hastie, 2013).

Una participación entusiasta del alumnado ha sido fre-

Fecha recepción: 18-10-18. Fecha de aceptación: 20-01-19

Federico Puente-Maxera

fedepuentem@hotmail.com cuentemente relacionada con la noción de disfrute, esfuerzo y motivación (Kinchin, Mac Phail, y Ni Chroinin, 2009), siendo percibido el MED como más atractivo respecto a metodologías basadas en la instrucción directa. Desde la perspectiva motivacional, numerosos estudios con el MED se han propuesto explicar las relaciones causales entre los aspectos que definen el modelo y los resultados de aprendizaje del alumnado (Chu y Zhang, 2018). La Teoría de la Autodeterminación (TAD; Deci y Ryan, 2000) sostiene que la motivación del individuo se mueve por un continuo gradual que va de menor a mayor autodeterminación, y señala a la motivación intrínseca como el nivel más deseado (Deci y Ryan, 2000). Desde este marco teórico la motivación en sí misma tiene su origen en la satisfacción de tres necesidades innatas que guían el comportamiento humano, denominadas necesidades psicológicas básicas (NPB). Así, existe la necesidad de autonomía, entendida como la posibilidad de actuar basándose en la elección y voluntad propias; competencia, referida a la necesidad de sentirse capaz y eficaz en el desarrollo de una tarea; y relación, que alude al deseo de establecer conexiones sociales positivas y recíprocas, así como sentirse aceptado por los demás (Deci y Ryan, 2000). Autores como Menéndez-Santurio y Fernández-Río (2017b) enfatizan la importancia de configurar espacios promotores de las NPB valiéndose para ello de modelos pedagógicos. En relación al MED, la evidencia muestra resultados positi- 
vos en cada una de estas dimensiones (Cuevas, García-López y Contreras, 2015; Perlman, 2011; Perlman, 2010), aspectos evidenciados en el contexto español, tal y como recoge la revisión de Evangelio, González-Víllora, Serra-Olivares, y Pastor-Vicedo, 2016).

El MED dispone de laxitud suficiente para ser adaptado a las necesidades del entorno (Kirk, 2013; Landi, Fitzpatrick y McGlashan, 2016). Desde su concepción original, el modelo ha sido testado en diferentes contextos culturales y socioeconómicos. Kirk (2013) indicó que la edad del alumnado constituye un aspecto clave en la selección del modelo pedagógico más apropiado. Las primeras revisiones sobre el MED (Hastie, Martínez de Ojeda y Calderón, 2011; Wallhead y O’Sullivan, 2005) presentan estudios abordados, mayormente, con estudiantes de educación secundaria. No obstante, en los últimos años, tal y como exponen Evangelio, Sierra-Díaz, Fernández-Rio, \& González-Víllora (2018) en su revisión, ha proliferado una gran producción en contextos de educación primaria (EP), tanto en tercer y cuarto curso (Calderón, Hastie y Martínez de Ojeda, 2010; Hastie, Ward, y Brock, 2017; Mowling, Brock y Hastie, 2006) como en quinto y sexto curso (Martínez de Ojeda, Calderón y Campos, 2012; Tsangaridou, y Lefteratos, 2013). Más escasas son las propuestas abordadas en los dos primeros cursos de EP (Layne, 2017; Layne y Hastie, 2016). Gutiérrez, García-López, Chaparro y Fernández (2014) examinaron el efecto del modelo en un grupo de segundo de EP (7-8 años) a partir de una temporada de balón prisionero. Los autores revelaron mejoras en el conocimiento del juego y en los patrones motrices, y destacaron la afiliación y la duración como aspectos claves del MED. Al mismo tiempo, advirtieron dificultades en el ejercicio de roles de mayor responsabilidad. Más recientemente, Layne y Hastie (2016) estudiaron la viabilidad de emplear el MED en un grupo de escolares de segundo curso de EP, informando de niveles apropiados de autonomía, así como una recepción entusiasta por parte del alumnado.

A pesar de estas valiosas evidencias (ambas sugiriendo la idoneidad del modelo con alumnado de edad temprana) hasta la fecha no se tiene conocimiento de trabajos publicados que analicen el impacto del MED en alumnado de primer curso de EP (6-7 años). Así pues, el propósito del presente estudio fue implementar el MED en un curso de primero de EP a efectos de conocer su impacto sobre las percepciones docentes y discentes respecto al modelo, así como sus efectos motivacionales (satisfacción de NPB). En torno a este objetivo, se establecieron como hipótesis que la intervención provocaría (i) aumentos significativos en cada uno de los tres objetivos que persigue el MED, así como (ii) mejoras en la satisfacción de cada una de las NPB.

\section{Método}

\section{Participantes y contexto}

En este estudio participaron 18 estudiantes (10 niños y ocho niñas) de primero de $\mathrm{EP}(M=6.61$ años; $D T=.50)$. Seis eran autóctonos, de nacionalidad española, y 12 inmigrantes, todos ellos de nacionalidad marroquí. Asimismo, participó en el estudio un docente especialista en Educación Física (EF) con una experiencia de 12 años, tres de ellos aplicando el MED. Nunca antes había experimentado el MED con estu- diantes de primero de EP. Para la implementación del estudio se contó con el consentimiento informado de las familias de los estudiantes y la autorización del centro escolar. El centro al que pertenecían el docente y los estudiantes a los que impartía EF estaba situado en una zona rural de la Región de Murcia (España).

\section{Diseño}

Se trata de un estudio de caso en el que se realizó un diseño cuasi experimental al objeto de analizar las percepciones tanto del docente como del alumnado. Se realizaron comparaciones con las siguientes variables independientes en los participantes: género y origen, intentando triangular los datos cualitativos y los cuantitativos. Estudios previos con el MED han seguido este tipo de metodologías (p.ej., Gutiérrez et al. 2014). Asimismo, destacan aquellos que se han servido de instrumentos cualitativos al afrontar muestras pequeñas como las del estudio actual (p.ej., Menéndez-Santurio, y Fernández-Río, 2017a).

\section{Unidad didáctica y validación}

El contenido de la unidad didáctica implementada fue el «pichi» (juego simplificado del béisbol). Su duración fue de 10 sesiones de 60 minutos, distribuidas en las siguientes fases: una sesión para la fase introductoria, dos sesiones para la fase dirigida, cuatro sesiones para la fase práctica, dos sesiones de competición formal y una sesión para el evento final (tabla 1). Aun no cumpliendo con la duración recomendada por la literatura (Siedentop et al. 2011), autores como García-López y Gutiérrez (2016, p. 100) aconsejan el abordaje de una «temporada exprés» (en torno a 10 sesiones) para experiencias iniciales.

\begin{tabular}{|c|c|}
\hline $\begin{array}{l}\text { SESIONES } \\
\text { (FASE) }\end{array}$ & CONTENIDO \\
\hline $1(\mathrm{I})$ & Explicación, formación de equipos, reparto de roles \\
\hline 2 (D) & Actividades de pase y recepción. \\
\hline 3(D) & $\begin{array}{l}\text { Reparto de zonas e iniciación de los roles específicos. Actividades de pase y } \\
\text { recepción. Golpeo de balón. Iniciación táctica (elección de zonas para } \\
\text { lanzamiento según ocupación del espacio, elección de la posta en la que parar, } \\
\text { elección de pase). }\end{array}$ \\
\hline $4(\mathrm{PR})$ & $\begin{array}{l}\text { Alumno-entrenador. Actividades de repaso. Otras actividades de pase y } \\
\text { recepción, y de golpeo de blón. Desarrollo táctico. }\end{array}$ \\
\hline $5(\mathrm{PR})$ & $\begin{array}{l}\text { Alumno-entrenador. Actividades de repaso. Otras actividades de pase y } \\
\text { recepción, y de golpeo de blón. Desarrollo táctico. }\end{array}$ \\
\hline $6(\mathrm{PR})$ & $\begin{array}{l}\text { Práctica autónoma. Explicación de la hoja de anotación. Partidos de práctica } \\
\text { con duty team. }\end{array}$ \\
\hline $7(\mathrm{PR})$ & Práctica autónoma. Partidos de práctica con duty team. \\
\hline 8 (CF) & Liga (3 partidos). Formato: triangular (ida) \\
\hline 9(CF) & Liga (3 partidos) Formato: triangular (vuelta) \\
\hline $10(\mathrm{EF})$ & $\begin{array}{l}\text { Entrega de diplomas y visionado de presentación multimedia de las vivencias } \\
\text { de los alumnos durante la unidad implementada. }\end{array}$ \\
\hline
\end{tabular}

Los equipos fueron seleccionados por el docente al objeto de que fueran heterogéneos en cuanto al nivel de habilidad, género y origen. Cada alumno asumió tres roles durante la temporada: (1) todos los alumnos fueron jugadores; (2) cada alumno asumió un rol específico (entrenador que fue elegido por el docente, encargado de material, encargado de salud y riesgo y preparador físico), que asumían en cada equipo durante toda la temporada; y (3) rol de duty team (árbitros y anotadores) de forma rotativa durante los partidos (tabla 2). De esta forma, durante la competición, un equipo ejercía de equipo organizador y los otros dos jugaban; al siguiente partido, rotaban. 


\begin{tabular}{|c|c|c|}
\hline Roles & Competición & $\begin{array}{l}\text { Sistema de } \\
\text { puntuación }\end{array}$ \\
\hline Jugador & $\begin{array}{l}\text { Regla 1. No podemos desplazarnos cuando tenemos el } \\
\text { balón en las manos, debemos pasarlo. }\end{array}$ & \\
\hline Salud y riesgo* & & Sumatorio de \\
\hline $\begin{array}{l}\text { E. Material* } \\
\text { Prep. Físico* }\end{array}$ & $\begin{array}{l}\text { por todos los conos) se consigue carrera. } \\
\text { Regla 3. El balón lanzado debe sobrepasar la línea de }\end{array}$ & $\begin{array}{l}\text { carreras } \\
\text { realizadas más }\end{array}$ \\
\hline Entrenador* & medio campo para que el lanzamiento sea válido. & puntos de fair \\
\hline Árbitro** & $\begin{array}{l}\text { Regla } 4 \text {. El jugador que hace de "pichi" no puede salir de la } \\
\text { zona indicada. }\end{array}$ & $\begin{array}{l}\text { play } \\
\text { conseguidos }\end{array}$ \\
\hline Anotador** & $\begin{array}{l}\text { Regla 5.- Si paramos en una base (cono) no podemos ser } \\
\text { eliminados. }\end{array}$ & \\
\hline
\end{tabular}

Además, se llevó a cabo una serie de medidas para implementar el MED en alumnos de esta edad (García-López y Gutiérrez, 2016). Estas adaptaciones (tabla 3) fueron revisadas y validadas por dos docentes de EP con más de 10 años de experiencia en $\mathrm{EF}$ en primero de $\mathrm{EP}$ y por dos doctores especialistas en metodología de la EF.

Tabla 3.

Características específicas de la implementación del MED en $1^{\circ} \mathrm{de} E P$.

Hoja de anotación Uso de pictogramas con texto muy breve.

Roles Una sola responsabilidad por rol, con la posibilidad de ampliar a una segunda a aquellos alumnos que aprendan completamente la primera.

Competición Selección de juego/deporte sencillo y con simplificación de reglas.

Alumno-entrenador Explicación muy breve de juegos muy sencillos. Ayuda gráfica de la explicación.

La validez de la implementación se hizo a través de la observación del investigador mediante una planilla traducida por Calderón et al. (2010) desde Sinelnikov (2009). De la planilla, tal y como sucedió en el estudio de Gutiérrez et al. (2014) no se planificaron ni observaron dos ítems: (1) el profesor da hojas de tareas diarias a los entrenadores; (2) el profesor incorpora la evaluación compartida como parte del proceso de recogida de datos.

\section{Instrumentos}

Entrevistas al docente. Se realizaron tres entrevistas semiestructuradas (Cohen y Manion, 2002) durante la experiencia (antes de empezar, a mitad de la implementación y al finalizarla) con el fin de conocer la percepción del docente (Calderón, et al., 2010). Los guiones fueron revisados por dos doctores y expertos en didáctica de la EF.

Diario del docente. El docente escribió, de forma libre, todas aquellas vivencias y aspectos que consideró relevantes acerca del proceso y de los alumnos, siguiendo las recomendaciones de Jurado (2011).

Entrevistas al alumnado. Una vez finalizada la intervención, los estudiantes fueron entrevistados dispuestos en grupos de seis (coincidiendo con los equipos establecidos en la unidad), tal y como recomiendan Ennis y Chen (2012). Se registraron tres entrevistas semi-estructuradas en las que los participantes respondieron a preguntas acerca de su experiencia a lo largo de la unidad. Un grupo de expertos revisó y ultimó los guiones de las entrevistas. Para llevar a cabo las entrevistas se utilizó un acompañamiento gráfico con fotografías de diferentes momentos de la unidad implementada, tal y como hicieron Layne y Hastie (2016) en su estudio con estudiantes de segundo de EP.

Dibujos argumentados. Al finalizar la experiencia, los alumnos realizaron un dibujo argumentado en el que debían dibujar aquello que reflejara la experiencia vivida con el MED. Tal y como hicieran Calderón et al. (2010, p. 174) se les dio las siguientes instrucciones: Piensa en la temporada de «ba- lón prisionero» que has vivido, y dibuja todo lo que pase por tu cabeza. En este caso su sustituyó «balón prisionero» por «pichi».

Cuestionario de Educación Deportiva. Al finalizar la unidad didáctica, los alumnos completaron el cuestionario del MED desarrollado por Mohr, Townsend, Rairigh y Mohr (2003) y posteriormente modificado por Hastie y Sinelnikov (2006) traducido al español por Calderón et al. (2010). Se trata de un cuestionario compuesto por seis ítems relacionados con las características del modelo y que se deben responder en una escala tipo Likert del 1 (nada) al 5 (mucho), y tres ítems (en los que el alumno debe responder su percepción antes de iniciar la unidad y al finalizarla) que se corresponden con los tres objetivos del MED y deben responderse en una escala tipo Likert del 1 (muy mal) al 10 (muy bien).

\section{Análisis de datos}

Entrevistas y diario. Una vez transcritas las entrevistas, estas y los diarios fueron analizados utilizando comparaciones constantes (Corbin y Strauss, 2008) y métodos de inducción analítica (Patton, 2002) con el objetivo de encontrar patrones comunes y poder identificar y agrupar la información en diferentes categorías. Tras una primera categorización, los datos fueron analizados de nuevo para contrastar los resultados encontrados (Miles y Huberman, 1994).

Dibujos argumentados. Los dibujos fueron analizados siguiendo los protocolos de análisis y las categorías indicadas por Mowling et al. (2006) y traducidas al español por Gutiérrez et al. (2014). Posteriormente, se calcularon los porcentajes para cada categoría.

Cuestionario del MED. Los datos cuantitativos fueron analizados a través del paquete estadístico IBM-SPSS (versión 20.0). La consistencia interna de las variables analizadas fue calculada mediante el coeficiente alfa de Cronbach, obteniendo niveles aceptables (á > .76). Asimismo, se solicitó la prueba de Shapiro-Wilk para valorar la normalidad del cuestionario, obteniéndose valores de Sig. < .05., por lo que se emplearon pruebas no paramétricas para la realización de los análisis pertinentes. En primer lugar, se calcularon los estadísticos descriptivos. Posteriormente, se empleó la prueba de los rangos con signo de Wilcoxon de medidas relacionadas introduciendo las medidas del pretest y posttest de los ítems relacionados con los tres objetivos del MED.

\section{Resultados}

\section{Entrevistas y diario}

Del análisis de las entrevistas y del diario realizados al docente y las entrevistas en grupo realizadas al alumnado se obtuvieron tres categorías: (1) planificación del MED; (2) entusiasmo de los estudiantes de primero de EP; (3) NPB, MED y edad de los estudiantes.

\section{Planificación del MED}

El docente indicó, a pesar de sus reticencias iniciales, que se cumplió lo planificado desde las primeras sesiones. En este sentido, indicó que para ello fue necesario una planificación «más exhaustiva, sin aspectos al azar, con todas las sesiones pormenorizadas». Conforme avanzaron las sesiones, el docente ratificó su postura inicial, aunque esto 
supone «mucho esfuerzo porque los niños están tardando un poco en aprender a realizar su rol» a pesar de que estos «estaban adaptados para primero de EP». Así, informó que la clave para que los alumnos realizaran bien su rol a esta edad era «ser muy repetitivo en las primeras sesiones», recordando constantemente sus responsabilidades y estando pendiente de las tareas a desempeñar.

Otro aspecto clave destacado por el docente fue el número de equipos a conformar: «he decidido realizar tres equipos por el número de componentes de cada equipo. De esta forma también consigo simplificar la complejidad a la hora de la competición formal, dos equipos juegan y el otro hace de duty team». Al respecto, los estudiantes fueron conscientes de la estructura de la competición: «teníamos alumnos que pitaban los partidos y otros que anotaban, mientras que los demás jugaban al pichi». En este sentido, al realizar los equipos el docente, se garantizó que estos fueran heterogéneos. La madurez del alumnado de primero de EP no aseguraba que, en una primera experiencia, pudieran realizarlos de forma adecuada. Además, señaló que el rol de entrenador es muy importante a la hora de conformar los equipos debido a que «la parte comunicativa es muy importante y se necesita a niños que puedan transmitir la información, por lo que en primero de EP es conveniente que los elija el docente». Así, el docente indicó la necesidad de partir de juegos conocidos por los estudiantes para las primeras intervenciones con el rol de entrenador y utilizar mensajes cortos y sencillos. A pesar de ello, en un principio, consideró que debería intervenir constantemente para que pudiera desarrollarse de manera conveniente: «yo creo que voy a tener que ayudar a los alumnos entrenadores», confirmando el esfuerzo que supone esta labor al acabar la experiencia: «la fase práctica ha sido más compleja que el resto porque a los niños les ha costado mucho entender la forma de trabajar; sin embargo, se ha conseguido». Para contrarrestar este esfuerzo en estas edades, el docente señaló que «se había diseñado una forma de transmitir esa información: primero tenía que agrupar a todos los niños y sentarlos delante de él, que dejasen todos los materiales, y después explicarles la actividad». Los estudiantes comentaron también la importancia de tener consignas para favorecer este proceso, tal y como afirmaba uno de los tres alumnos con rol de entrenador: «yo sentaba a mis compañeros para que me escucharan y les decía lo que me había dicho el maestro».

Los roles de duty team formaron parte también de la preocupación del docente al planificar la unidad. Así, indicó la necesidad de simplificar la dificultad de la hoja de anotación y las reglas, aspecto este último que contribuyó a la elección del contenido por parte del docente, el pichi, un contenido con «unas reglas sencillas». Conforme se desarrollaron las sesiones percibió que «el desempeño del duty team ha sido bastante complejo», sobre todo la parte del arbitraje la cual «les ha costado más». Como aspecto importante a la hora de planificar una unidad en primero de EP consideró que «habría que darle una vuelta de tuerca al arbitraje», refiriéndose a la forma de aprenderlo alumnos de corta edad.

En línea con todo lo anteriormente argumentado por el docente, este señaló que desaconsejaba una primera experiencia con primero de EP:

«Desaconsejaría que la primera experiencia del do- cente con el MED fuera con primero de EP. No sé cómo me va a salir, espero que bien, pero si no me sale bien no me voy a desanimar porque ya lo he visto funcionar antes. Pero si lo hace alguien sin experiencia previa con el modelo en otros cursos y no le sale bien puede pensar que este modelo no funciona».

En este mismo sentido, concluyó que «para una primera experiencia del docente aconsejaría cursos altos de EP, dejando a los cursos más bajos para cuando tuviera cierta experiencia con el modelo».

Así, aconsejó que, si se aplicaba el modelo en primero, además de la experiencia previa con el mismo, era necesario realizarlo, al menos, en el segundo trimestre, desaconsejando totalmente su implementación en el primero: «con primero es mejor no realizar la unidad didáctica con el modelo al inicio de curso porque tienen que hacer ya una adaptación compleja en el trabajo en EF al subir desde la etapa de educación infantil».

\section{Entusiasmo de estudiantes de primero de EP}

El docente se mostró muy optimista con respecto al entusiasmo del alumnado durante la unidad didáctica. Así, antes de la implementación de la unidad indicó: «creo que les va a gustar mucho, les va a encantar». Estos pronósticos los confirmó según avanzó la temporada:

«Los niños de primero están muy motivados siempre con la EF. Dentro de la EF hay juegos que les gusta más y juegos que les gusta menos. Cuando a los niños de primero no les gusta un juego desconectan y se ponen a hacer otra cosa y ahora ocurre lo contrario, la forma de trabajar y todos los juegos que hacen les parecen interesantes y creo que es por la asignación de roles, la autonomía y porque cada uno tiene un papel importante dentro del equipo; no solo por el hecho de estar jugando».

Concretamente, señaló la implementación de los roles asignados como un elemento clave en este desarrollo del entusiasmo: «uno de los aspectos más destacados es la motivación del alumnado. Los niños tienen mucha ilusión por hacer bien su rol, por formar parte de un equipo $y$ sentirse una pieza útil dentro de la clase de EF».

En esta misma línea, diferentes alumnos y alumnas señalaron que asumir roles con responsabilidades que cumplir, les entusiasmó. Diferentes estudiantes hicieron alusión a esto comentando... «los roles nos gustaron mucho». A pesar de ello, hubo algún comentario contrario. Un alumno señaló que «no me ha gustado que hubiera compañeros que me dijeran lo que tenía que hacer».

El docente vaticinó que el evento final sería uno de los aspectos más relevantes en el entusiasmo del alumnado: «El evento final les va a hacer mucha ilusión, aunque no sé cómo se lo van a tomar los que se queden en tercer lugar (últimos)». Este aspecto lo ratificó al concluir la unidad: «El evento final resultó bastante bonito, fue todo un éxito. Fue muy motivante para los niños ver el trabajo que habían hecho durante toda la unidad por equipos y además recibir un diploma. Fue muy ilusionante para ellos y para mí».

Asimismo, a pesar de pensar en un principio que «a niños tan pequeños les cansaría estar jugando tantos días a lo mismo», al finalizar indicó que ocurrió todo lo contrario; 
los niños le pedían seguir jugando: «A pesar de ser la unidad didáctica más larga que han realizado este año no se cansaron, todo lo contrario, pedían seguir. De hecho, si hubiera tenido más sesiones habría salido aún mejor». En esta misma línea, el docente expresó que los niños también deseaban seguir trabajando bajo la misma estructura de unidad: «al terminar la unidad los niños me pedían seguir trabajando de la misma forma y contar con sus roles y responsabilidades».

Los alumnos también reportaron haber vivenciado una experiencia divertida. Así, diferentes alumnos y alumnas indicaron: «Nos ha parecido corta la unidad, nos gustaría seguir jugando». Además, el hecho de trabajar con el MED supuso un aumento en el entusiasmo: «nos ha gustado más trabajar así». Asimismo, reportaron el trabajo en equipo como la clave para que la experiencia haya sido divertida y les haya gustado. En este sentido, hubo numerosos comentarios del alumnado: «nos gusta trabajar en equipo porque nos divertimos más; o me ha gustado mucho estar con mi equipo».

\section{NPB, MED y edad}

Desde un inicio el docente confió en que el alumnado mejoraría tanto la técnica como la táctica, fundamentalmente, por el trabajo en equipo: «van a mejorar sobre todo el trabajo de equipo... la idea de poder contar con compañeros para conseguir un objetivo común y llegar a tener conciencia de que saldrá mejor que si lo hago de forma individual». Sin embargo, conforme avanzó la unidad empezaron a aparecer dudas con respecto a la competencia que tendrían los alumnos para desarrollar las labores de duty team y cómo influiría esto en lo que restaba de unidad y, por ende, en el desarrollo competencial del alumno. Así, el docente indicó que «la competición va a depender de la fase en la que se aprende a ser duty team, que aprendan las reglas y aprendan a pitar, y son niños de seis años por lo que no sé si serán capaces de realizar bien el duty team». Al finalizar la implementación comentó que los alumnos habían mejorado mucho disipando así las dudas iniciales: «a nivel motriz y a nivel técnico les ha venido muy bien, y a nivel táctico les ha venido genial; han aprendido a lanzar lejos de donde está el resto de niños para que no puedan coger la pelota pronto». En este sentido, los alumnos tuvieron la sensación de haber realizado bien las labores de anotación y de resultarles más complicadas las propias del arbitraje, tal y como comentó un niño: «nos ha resultado fácil anotar, pero pitar ha sido regular (sic)».

Por otro lado, el docente subrayó que las dudas iniciales se debían a la falta de autonomía propia de la edad del alumnado. En este sentido, al inicio, indicó que debía de estar pendiente de cada aspecto: «al principio tuve que acotar las zonas con conos para que tuviesen claro los límites de las zonas de trabajo». No obstante, conforme avanzaron las sesiones informó de la mejora de autonomía del alumnado: «en estas sesiones ya están siendo más dinámicos y más autónomos y de momento están funcionando solos», destacando la figura del alumno-entrenador como reflejo de esta mejora:

«La sorpresa ha venido en la primera sesión que he tenido con alumno entrenador, donde tras llamar a los entrenadores para explicar las tareas a transmitir a sus compañeros han sido capaces de sentar a sus compañeros y explicarles juegos que ya habían hecho y juegos tácticos que no habían practicado nunca y que son relativamente complicados a la hora de organizar».

En este sentido, los alumnos llegaron a sentirse cómodos con la figura de entrenador asegurando que «al final era fácil transmitir la información a mis compañeros». En esta misma línea, diferentes alumnos argumentaron el respeto a esta figura y que ello supuso una percepción positiva de la experiencia. Un alumno con el rol de entrenador comentó: «mi equipo ha trabajado muy bien y me ha escuchado muy bien».

Al finalizar la unidad el docente comentó que «son niños de 6 años. La autonomía total es complicada, no solo en esta actividad sino en cualquier otra, aun así, yo he tenido que intervenir muy poco. Ellos han sido capaces de gestionar el juego, de gestionar el arbitraje y la anotación». A pesar de ello, concluyó que

«El nivel de autonomía empieza a ser parecido a los cursos mayores que utilizan este modelo. Yo solo intervengo cuando me lo pide el entrenador porque tiene algo que resolver dentro del equipo o para transmitir la información al alumno entrenador, nada más. Antes eran totalmente dependientes a la hora de resolver un problema, a la hora de realizar un ejercicio, en casi todos los aspectos eran dependientes».

Esta autonomía, según expresó el docente, empezó a ser notable en la fase de competición. Así, indicó que «al principio esperaban a que el maestro dijese la última palabra a la hora de arbitrar, si había entrado el jugador o no. Ahora no, ahora son capaces por sí solos de saber, por ejemplo, si está eliminado el jugador o no, y casi no intervengo».

Esta mejora de la autonomía se reflejó también en la capacidad de resolver problemas:

«Durante la práctica de la unidad didáctica los niños eran mucho más autónomos, sobre todo en el aspecto de resolver sus propios problemas hablando y llegando a acuerdos en el equipo. Cuando era de forma tradicional solo estaba la figura del maestro para resolver los problemas, eran incapaces».

En este sentido, el docente, al inicio, argumentó que aunque «son niños muy pequeños y un poco imprevisibles creo que van a tener una buena afiliación en sus equipos y no va a haber ningún problema significativo», se trata de «niños de seis años y la capacidad de autocontrol la tienen regular todavía». Al finalizar la experiencia, la afiliación al equipo fue clave, a los ojos del docente, para la mejora en las relaciones sociales:

«No se plantean estar en otro equipo, es decir, están en su equipo y es con ellos con los que van hacia delante y están motivados hasta el punto de que ellos tienen un grito que es el que se realiza antes de iniciar los partidos; sin embargo, de forma espontánea, lo están haciendo cuando terminan el calentamiento».

Los alumnos señalaron el trabajo en equipo como aspecto clave para la mejora tanto de la competencia como de las relaciones sociales. Así, indicaron que «hemos aprendido a jugar al pichi porque lo hemos hecho entre todos o no ha habido problemas, las cosas las decidíamos todo el equipo». 


\section{Dibujos argumentados}

Tal y como se recoge en la tabla 4, el alumnado expresó, en sus dibujos argumentados, un alto nivel de afiliación a través de expresiones artísticas como la incorporación de todos los componentes del equipo o colores identificativos. Asimismo, fue unánime la manifestación de felicidad en todos los dibujos. Por otro lado, la figura del docente no fue reflejada en ninguno de los dibujos realizados. Sin embargo, ningún dibujo hizo alusión a una de las características del MED, el duty team, y ningún dibujo incorporó la figura de árbitro o anotador. Por último, el mayor porcentaje de expresiones relacionadas con la competición se dio en los chicos y, según la procedencia, en los autóctonos.

\begin{tabular}{|c|c|c|c|c|c|}
\hline & TOTAL & CHICAS & CHICO & ESP. & INM. \\
\hline Dibuja a otros compañeros (DOC) & 100 & 100 & 100 & 100 & 100 \\
\hline Se dibuja "dentro" del juego (DDJ) & 55.56 & 50 & 60 & 66.67 & 50 \\
\hline Equipo contrario (EC) & 16.67 & 0 & 30 & 33.33 & 8.33 \\
\hline Desarrollo habilidades de equipo (DHE) & 27.78 & 37.5 & 20 & 33.33 & 25 \\
\hline Emociones (feliz) (E[fl]) & 100 & 100 & 100 & 100 & 100 \\
\hline Partidos competición (PC) & 22.22 & 0 & 40 & 33.33 & 16.67 \\
\hline Un balón (B) & 55.56 & 37.5 & 70 & 83.33 & 41.67 \\
\hline Conos (C) & 33.33 & 37.5 & 30 & 50 & 25 \\
\hline Nombre equipo (NE) & 22.22 & 12.5 & 30 & 33.33 & 16.67 \\
\hline Nombre del juego (NJ) & 16.67 & 25 & 10 & 16.67 & 16.67 \\
\hline Desarrollo habilidades individuales (DHI) & 0 & 0 & 0 & 0 & 0 \\
\hline Gimnasio $(\mathrm{G})$ & 61.11 & 62.5 & 60 & 50 & 66.67 \\
\hline Puntuación final (PF) & 0 & 0 & 0 & 0 & 0 \\
\hline Marcador (M) & 0 & 0 & 0 & 0 & 0 \\
\hline Ausencia de desarrollo de habilidades (ADH) & 72.22 & 62.5 & 80 & 66.67 & 75 \\
\hline Compañeros de equipo (CE) & 100 & 100 & 100 & 100 & 100 \\
\hline Entrega de diplomas (ED) & 0 & 0 & 10 & 16.67 & 0 \\
\hline Se dibuja a sí mismo (DAM) & 100 & 100 & 100 & 100 & 100 \\
\hline Grupo completo (GC) & 61.1 & 50 & 70 & 66.67 & 66.67 \\
\hline Desarrollo habilidades de clase (DHC) & 0 & 0 & 0 & 0 & 0 \\
\hline Profesores (AP/MC) & 0 & 0 & 0 & 0 & 0 \\
\hline Varios balones (VB) & 0 & 0 & 0 & 0 & 0 \\
\hline Entrenamientos (E) & 27.78 & 37.5 & 20 & 33.33 & 25 \\
\hline Premios (P) & 0 & 0 & 0 & 0 & 0 \\
\hline Emociones (éxito) (E[é]) & 11.11 & 12.5 & 10 & 33.33 & 0 \\
\hline Evento final (EF) & 5.56 & 0 & 10 & 16.67 & 0 \\
\hline Referencias al árbitro (RA) & 0 & 0 & 0 & 0 & 0 \\
\hline Colores del equipo (CDE) & 83.33 & 87.5 & 80 & 83.33 & 83.33 \\
\hline Anotadores (An) & 0 & 0 & 0 & 0 & 0 \\
\hline Mensajes de ánimo (MA) & 0 & 0 & 0 & 0 & 0 \\
\hline Se dibuja "fuera" del juego (DFJ) & 0 & 0 & 0 & 0 & 0 \\
\hline Árbitros (Ar) & 5.56 & 0 & 10 & 16.67 & 0 \\
\hline Emociones (fracaso)(E[fr]) & 0 & 0 & 0 & 0 & 0 \\
\hline Emociones (triste) $(\mathrm{E}[\mathrm{t}])$ & 0 & 0 & 0 & 0 & 0 \\
\hline Emociones (nada) (E[n]) & 0 & 0 & 0 & 0 & 0 \\
\hline Amistosos (AM) & 0 & 0 & 0 & 0 & 0 \\
\hline $\begin{array}{l}\text { Desarrollo habilidades estratégicas del equipo } \\
\text { (DHEsE) }\end{array}$ & 0 & 0 & 0 & 0 & 0 \\
\hline Emociones (como “loco") (E[1]) & 0 & 0 & 0 & 0 & 0 \\
\hline Pretemporada (Pr) & 0 & 0 & 0 & 0 & 0 \\
\hline
\end{tabular}

En la figura 1 puede verse un ejemplo de dibujo y categorización del mismo.

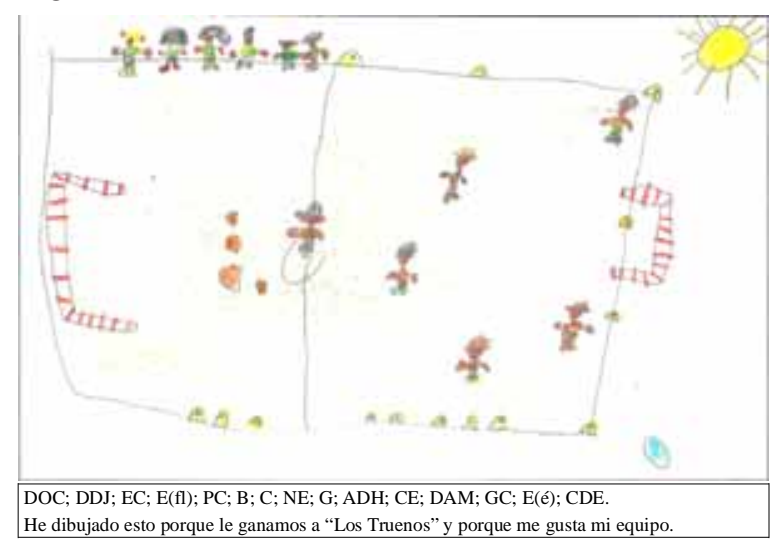

Figura 1. Ejemplo de dibujo categorizado y comentario realizado por un alumno.

\section{Cuestionario de Educación Deportiva}

Las características del modelo (tabla 5) fueron valoradas por encima de 4 puntos sobre 5 , siendo la duración, la afiliación y la fase final las que recibieron mayor puntuación.
Respecto al género, las chicas puntuaron más alto todas las características, salvo el registro. Y, respecto al origen, los inmigrantes valoraron más alto la afiliación, la estructura y la festividad, mientras que los autóctonos valoraron más la duración, la fase final y el registro de datos.

En cuanto a los objetivos del MED (tabla 6), los valores postest fueron en todos los casos más elevados que los pretest, existiendo diferencias significativas en entusiasmo $(Z=-2.23 ; p=.03)$ y cultura deportiva $(Z=-2.56 ; p=.011)$ de forma general, y en cultura deportiva en los inmigrantes $(Z=$ $-2.23 ; p=.03)$.

Tabla 5.

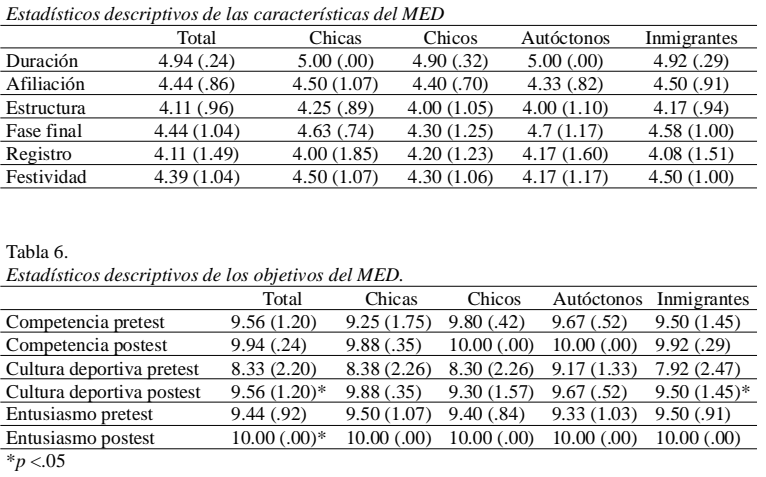

\section{Discusión}

El presente estudio se propuso implementar el MED en un grupo de primero de EP y analizar su repercusión sobre la percepción docente y discente respecto al modelo, así como en variables motivacionales. En general, los hallazgos del presente estudio convergen con los de estudios previos realizados en segundo curso de EP (Gutiérrez, et al., 2014; Layne y Hastie, 2016). Los resultados cuantitativos y cualitativos sugieren la aptitud del modelo para ser introducido desde un primer momento de la EP y desvelan, a su vez, una serie de aspectos didácticos a ser considerados para su abordaje en dicha fase. En primer lugar, la intervención provocó mejoras significativas en dos de los objetivos perseguidos por el MED, confirmando parcialmente la hipótesis inicial. La cultura deportiva fue la dimensión que experimentó mayores cambios significativos, dada su incidencia específica sobre el alumnado de origen inmigrante. Entre las razones, la exposición a roles distintos al de jugador/a (p.ej., arbitraje) pudo incidir en el desarrollo de una mejor comprensión del juego (Calderón, et al., 2010; Hastie y Sinelnikov, 2006; Hastie, et al., 2009). Partir de juegos conocidos, así como simplificar sus reglas, supusieron dos estrategias eficaces con alumnado de esta edad.

Igualmente, la intervención promovió un aumento significativo en la percepción general de entusiasmo, alcanzando la máxima puntuación posible. Un aspecto reseñable estribó en la alusión a emociones de felicidad (Calderón, et al., 2013; Mowling, et al., 2006), circunstancia unánime en el estudio actual. Varias razones pueden explicar una mayor participación entusiasta. Primero, la afiliación ha sido asociada a una mayor diversión (MacPhail, Gorely, Kirk, y Kinchin, 2008) redundando en deseos por mantener la forma de trabajo. Segundo, el reclamo discente de un mayor número de sesiones sugiere que la duración la unidad entusiasmó al alumnado 
(Calderón, et al., 2010; Gutiérrez, et al., 2014). El evento final también fue percibido como ilusionante y motivante (Hastie y Sinelnikov, 2006; Kinchin, et al., 2009) pudiendo incidir también en los niveles de entusiasmo. Cabe destacar que estos tres aspectos (afiliación, duración y evento final) fueron los más valorados por los estudiantes. Por su parte, la asunción de roles, si bien fue genéricamente descrita como positiva (Layne y Hastie, 2016), también provocó sensaciones negativas en parte del alumnado motivadas por recibir indicaciones de sus pares.

Desde la perspectiva cualitativa se advirtieron efectos positivos en la competencia del alumnado. El trabajo en equipo fue expuesto como causante de mejoras en el dominio técnico-táctico (Hastie y Trost, 2002). No obstante, a nivel cuantitativo, la ausencia de cambios significativos pudo deberse a razones diversas. En primer lugar, y en línea con lo expuesto por Calderón et al. (2010), parece constatarse el mayor impacto en el entusiasmo, en relación al objetivo competencial, durante la etapa de EP respecto a la secundaria. En segundo lugar, las puntuaciones previas fueron muy elevadas $(M=9.56)$ pudiendo provocar que la curva de aprendizaje estuviese limitada por un «efecto techo». Finalmente, el desempeño de ciertos roles pudo acarrear efectos nocivos en el desarrollo competencial. Las perspectivas docente y discente convinieron en informar de la dificultad del rol arbitral, siendo un hecho previamente advertido (Gutiérrez et al., 2014; Hastie y Sinelnikov, 2006). Los resultados derivados de las ilustraciones apuntan que el rol arbitral no despertó gran interés para el alumnado de esta edad. Mowling et al. (2006) reflexionaron sobre la inclusión de dicho rol o bien en etapas educativas más avanzadas o con alumnado conocedor del modelo. A su vez, cabe destacar que el registro fue, junto a la estructura, el elemento menos valorado por el alumnado. Considerando las recomendaciones previas (Gutiérrez et al., 2014), se debe cuidar este rol en escolares de edad temprana. No obstante, la labor de anotación fue desempeñada con suficiencia (Layne, y Hastie, 2016), siendo recomendada la simplificación de la hoja de anotación.

De especial notoriedad fue la incidencia de la intervención sobre el nivel de autonomía; un hecho sumamente apreciado, habida cuenta de la dificultad para promover comportamientos autónomos en esta edad (Chung, 2000), así como los complejos procesos de transición inherentes a la etapa. La omisión unánime de la figura del profesor en las ilustraciones dio cuenta del escaso papel hegemónico del docente, tal como sucedió en estudios previos (Calderón, et al., 2010; Gutiérrez, et al., 2014). Junto a la asunción de roles, los niveles de autonomía parecieron verse reforzados por varios aspectos. Consistente con Layne y Hastie (2016), se corroboró la conveniencia de priorizar los aspectos organizativos durante las primeras sesiones. Gutiérrez et al. (2014) insistieron en el tiempo requerido para el aprendizaje del modelo en estas edades. Asimismo, la estructura de las tareas, con consignas claras, sencillas y asumibles por el alumnado, favoreció el funcionamiento autónomo. Esto último repercutió sobre los roles de mayor centralidad destacándose su grado de cumplimiento durante las fases de mayor sensibilidad (p.ej., duty team en fase de competición; alumno entrenador durante la práctica autónoma). En este sentido, varios autores comparten la idea de incluir periodos de formación específi- ca para este tipo de roles (Farias, Hastie y Mesquita, 2017), y de forma imperativa en edades iniciales (Calderón, et al., 2010).

Finalmente, la organización social repercutió sobre la necesidad de relación (Perlman, 2010). Entre las situaciones didácticas más distinguidas, la persecución de un objetivo común supuso un avance en la interacción social. Asimismo, el alumnado mostró conformidad respecto a la composición de los equipos. En aras de garantizar heterogeneidad, la literatura previa (Gutiérrez, et al. 2014) sugiere que en edades iniciales esta deba estar relegada al docente, aspecto contemplado en el estudio actual. Asimismo, se vislumbró la eficacia sobre la relación entre iguales de un elemento propio de la festividad, el grito de equipo que, por iniciativa propia, los estudiantes de este estudio extendieron a la fase de calentamiento. En su estudio, Hastie y Sinelnikov (2006) observaron que el alumnado hizo extensible este elemento a los tiempos de recreo. Desde la perspectiva discente, se destacó la importancia dada por este alumnado al color de equipo, siendo desvelado como otro elemento esencial de cohesión grupal y relación entre iguales (Tsangaridou y Lefteratos, 2013).

\section{Conclusiones}

Los resultados apuntan que el MED pueda ser aplicado con éxito en toda la etapa de EP, incluso con estudiantes más pequeños. Se destaca el impacto positivo de varios de los elementos que le caracterizan sobre las NPB, ya sea la afiliación (competencia), los roles (autonomía) o elementos festivos (relación entre iguales). Sin embargo, es necesario tener en consideración una serie de recomendaciones para el primer curso: (1) es aconsejable que el docente tenga experiencia previa con el MED en cursos superiores; (2) es necesario adaptar algunos materiales al nivel cognitivo y comprensivo de los niños de estas edades (p.ej., hoja de anotación o responsabilidades de los roles); (3) se deben simplificar las reglas del juego, y (4) se debe iniciar el MED a partir del segundo o tercer trimestre.

El presente estudio cuenta con una serie de limitaciones. Primero, el propio diseño de investigación, carente de grupo control y materializado en un corto periodo de tiempo, provoca resultados difícilmente extrapolables. Segundo, la concreción del estudio a una única clase y un único docente, hace necesaria su réplica en muestras de mayor tamaño, así como en contextos de diferentes características (culturales, socioeconómicas, etc.). Finalmente, será de interés el análisis de variables no contempladas en este estudio (como, p.ej., la percepción de habilidad física).

\section{Referencias}

Calderón, A., Hastie, P. A., y Martínez de Ojeda, D. (2010). Aprendiendo a enseñar mediante el modelo de Educación Deportiva. Experiencia inicial en Educación Primaria. Cultura, Ciencia y Deporte, 5, 169-180.

Calderón, A., Martínez de Ojeda, D., y Hastie, P. A. (2013). Valoración de alumnado y profesorado de Educación Física tras la aplicación de dos modelos de enseñanza. RICYDE. Revista internacional de ciencia y deporte, 32(9), 137-153. 
Chu, T. L., y Zhang, T. (2018). Motivational processes in Sport Education programs among high school students: A systematic review. European Physical Education Review, 20(10), 1-23.

Chung, M. (2000). The development of self-regulated learning. Asia Pacific Education Review, 1(1), 55-66.

Cohen, L., y Manion, L. (2002). Métodos de investigación educativa. Madrid: La Muralla.

Corbin, J. M., y Strauss, A. L. (2008). Basics of Qualitative Research: Techniques and Procedures for Developing Grounded Theory. Los Angeles, CA: Sage publications.

Cuevas, R., García-López, L. M., y Contreras, O. (2015). Influencia del modelo de Educación Deportiva en las necesidades psicológicas básicas. Cuadernos de Psicología del Deporte, 15(2), 155162.

Deci, E. L., y Ryan, R. M. (2000). The «what» and «why» of goa pursuits: Human needs and the self-determination of behaviour. Psychological Inquiry, 11, 227-268, doi: 10.1207/ S15327965PLI1104 01

Ennis, C. D., y Chen, S. (2012). Interviews and focus groups. En K. Armour y D. Macdonald (Eds.), Research Methods in Physical Education and Youth Sport, 217-236. New York: Routledge.

Evangelio, C., González-Víllora, S., Serra-Olivares, J. y Pastor-Vicedo, J.C. (2016). El Modelo de Educación Deportiva en España: una revisión del estado de la cuestión y prospectiva. Cuadernos de Psicología del Deporte, 16(1), 307-324.

Evangelio, C., Sierra-Díaz, J. M., Fernández-Rio, J. y González-Víllora, S. (2018). Sport education model in elementary and secondary education: Systematic review. Movimento, 24(3), 931-946.

Farias, C., Hastie, P. A., y Mesquita, I. (2017). Scaffolding studentcoaches' instructional leadership toward student-centred peer interactions: A yearlong action-research intervention in sport education. European Physical Education Review, 1-23.

García-López, L. M., y Gutierrez, D. (2016). Aprendiendo a enseñar deporte. Modelos de enseñanza comprensiva y educación deportiva. Barcelona: INDE.

García-López, L. M., Gutiérrez, D., González-Víllora, S., y Valero, A (2012). Cambios en la empatía, la asertividad y las relaciones sociales por la aplicación del modelo de instrucción educación deportiva. Revista de Psicología del Deporte, 2, 321-330.

Gutiérrez, D., García-López, L. M., Chaparro, R., y Fernández, A. J. (2014). Aplicación del modelo de Educación Deportiva en segundo de Educación Primaria: percepciones del alumnado y el profesorado. Cuadernos de Psicología del Deporte, 14(2), 131144

Hastie, P. A., Martínez de Ojeda, D., y Calderón, A. (2011). A review of research on Sport Education: 2004 to the present. Physical Education \& Sport Pedagogy, 16(2), 103-132.

Hastie, P. A., y Sinelnikov, O. A. (2006). Russian students' participation in and perceptions of a season of Sport Education. European Physical Education Review, 12(2), 131-150.

Hastie, P. A., Sinelnikov, O. A., y Guarino, A. J. (2009). The development of skill and tactical competencies during a season of badminton. European Journal of Sport Science, 9, 133-140.

Hastie, P. A., y Trost, S. G. (2002). Student physical activity levels during a sport education season. Pediatric Exercise Science, 14(1), 64-74.

Hastie, P. A., y Wallhead, T. (2016). Models-Based Practice in Physical Education: The Case for Sport Education. Journal of Teaching in Physical Education, 35, 390-399.

Hastie, P. A., Ward, K. y Brock, S. (2017). Effect of graded competition on student opportunities for participation and success rates during a season of Sport Education. Physical Education \& Sport Pedagogy, 22(3), 316-327.

Jurado, M. D. (2011). El diario como instrumento de autoformación e investigación. Revista Qurriculum, 24, 173-200.

Kinchin, G. D., MacPhail, A., y Ni Chroinin, D. (2009). Pupils’ and teachers' perceptions of a culminating festival within a sport education season in Irish primary schools. Physical Education \& Sport Pedagogy, 14(4), 391-406. doi: 10.1080/ 17408980802584982

Kirk, D. (2013). Educational value and models-based practice in physical education. Educational Philosophy and Theory, 45, 973-986.

Landi, D., Fitzpatrick, K., y McGlashan, H. (2016). Models Based Practices in Physical Education: A Sociocritical Reflection. Journal of Teaching in Physical Education, 35(4), 400-411. doi:10.1123/jtpe.2016-0117.

Layne, T. (2017). Sport Education in the primary grades. ACHPER Active and Healthy Journal, 24, 44-47.

Layne, T., y Hastie, P. A. (2016). Analysis of teaching physical education to second-grade students using sport education. Education 3-13, 44(2), 226-240.

MacPhail, A., Gorely, T., Kirk, D., y Kinchin, G. D. (2008). Children’s experiences of fun and enjoyment during a season of Sport Education. Research Quarterly for Exercise and Sport, 79(3), 344-355.

Mahedero, M. P., Calderón, A., Arias, J. L., Hastie, P. A., y Guarino, A. (2015). Effects of student skill level on knowledge, decision making, skill execution and game performance in a mini-volleyball sport education season. Journal of Teaching in Physical Education, 34, 626-641. doi:10.1123/jtpe.2014-0061

Martínez de Ojeda, D., Calderón, A., y Campos, A. (2012). Percepción de aprendizaje y satisfacción en una unidad didáctica integrada mediante el modelo de educación deportiva. Cultura, Ciencia y Deporte, 7(21), 163-172.

Méndez-Giménez, A., Fernández-Río, J., y Méndez-Alonso, D. (2015). Modelo de educación deportiva versus modelo tradicional: efectos en la motivación y deportividad. Revista Internacional de Medicina y Ciencias de la Actividad Física y el Deporte, 15(59), 449-466.

Menéndez-Santurio, J. I., y Fernández-Río, J. (2017a). Hybridizing Sport Education and Teaching for Personal and Social Responsibility to include students with disabilities. European Journal of Special Needs Education, 34(2), 508-524.

Menéndez-Santurio, J. I., y Fernández-Río, J. (2017b). Responsabilidad social, necesidades psicológicas básicas, motivación intrínseca y metas de amistad en educación física. Retos. Nuevas Tendencias en Educación Física, Deporte y Recreación, 32, 134-139.

Metzler, M. W. (2011). Instructional models for physical education ( $2^{\mathrm{a}}$ ed.). Scottsdale, AZ: Holcomb Hathaway Publishing.

Miles, M. B., y Huberman, A. M. (1994). Qualitative data analysis: An expanded sourcebook. Thousand Oaks, CA: Sage Publications.

Mohr, D. J., Townsend, J. S., Rairigh, R., y Mohr, C. (2003). Students' perceptions of Sport Education when taught using the pedagogical approach to Sport Education (PASE) planning and instructional framework. Research Quarterly for Exercise and Sport, 74, A51.

Mowling, C. M., Brock, S. J., y Hastie, P. A. (2006). Fourth grade students' drawing interpretations of a sport education soccer unit. Journal of Teaching in Physical Education, 25, 9-35.

Patton, M. Q. (1990). Qualitative evaluation and research methods. Newbury Park, CA: Sage.

Perlman, D. (2010). Change in affect and needs satisfaction for amotivated students within the sport education model. Journal of Teaching in Physical Education, 29(4), 433-445.

Perlman, D. (2011). Examination of self-determination within the Sport Education Model. Asia-Pacific Journal of Health, Sport and Physical Education, 2(1), 79-92.

Siedentop, D. (1994). Sport education: Quality PE through positive sport experiences. Champaign, IL: Human Kinetics.

Siedentop, D., Hastie, P. A., y van der Mars, H. (2011). Complete Guide to Sport Education ( $2^{\mathrm{a}}$ ed.). Champaign, IL: Human Kinetics.

Sinelnikov, O. A. (2009). Sport education for teachers: Professional development when introducing a novel curriculum model. European Physical Education Review, 15, 91-114.

Tsangaridou, N., y Lefteratos, C. (2013). Elementary students’ views and experiences on sport education in Cyprus. Advances in Physical Education, 3, 28-35. doi:10.4236/ape.2013.31005

Wallhead, T., y O’Sullivan, M. (2005). Sport education: Physical education for the new millennium? Physical Education \& Sport Pedagogy, 10(2), 181-210. 